Revue de droit comparé du travail et de la sécurité sociale

$3 \mid 2019$

Les migrations internationales de travail

\title{
Réforme du financement des syndicats
}

Ana Virginia Moreira Gomes et Gabriella de Assis Wanderley

\section{OpenEdition}

Journals

Édition électronique

URL : https://journals.openedition.org/rdctss/1507

DOI : $10.4000 /$ rdctss. 1507

ISSN : 2262-9815

Éditeur

Centre de droit comparé du travail et de la sécurité sociale

Édition imprimée

Date de publication : 1 novembre 2019

Pagination : 156-159

ISSN : 2117-4350

Référence électronique

Ana Virginia Moreira Gomes et Gabriella de Assis Wanderley, «Réforme du financement des

syndicats », Revue de droit comparé du travail et de la sécurité sociale [En ligne], 3 | 2019, mis en ligne le 01 novembre 2021, consulté le 13 novembre 2021. URL : http://journals.openedition.org/rdctss/1507 ; DOI : https://doi.org/10.4000/rdctss. 1507 Licence Creative Commons Attribution - Pas d'Utilisation Commerciale - Pas de Modification 4.0 International. 


\section{ANA VIRGINIA MOREIRA GOMES GABRIELLA DE ASSIS WANDERLEY}

UNIVERSITÉ DE FORTALEZA

\section{RÉFORME DU FINANCEMENT DES SYNDICATS}

La loi brésilienne $n^{0} 13.467$ sur la réforme du travail, promulguée le 13 juillet 2017, a rendu facultatives les cotisations syndicales corporatistes obligatoires depuis 1931 (contribuição sindical) en modifiant l'article 578 de la Consolidation des lois du travail (CLT). Le motif politique de ce changement était d'éliminer une cotisation universelle imposée par la loi, aboutissant finalement à des syndicats complaisants et souvent non représentatifs. Quant à la motivation légale, elle résidait dans l'incompatibilité entre la cotisation obligatoire et la dimension négative de la liberté d'association, c'est-à-dire la liberté de ne pas adhérer à un syndicat ou de s'en dissocier.

La suppression du caractère obligatoire des cotisations syndicales a été jugée constitutionnelle en 2018 par la Cour suprême fédérale (STF)․ Ce changement normatif soulève une question importante pour le droit du travail brésilien. Au Brésil, les syndicats représentent les droits et les intérêts des travailleurs et des employeurs regroupés en catégories ${ }^{2}$. Tous sont représentés, qu'ils aient ou non adhéré volontairement au syndicat. Dans le système établi par la loi $n^{\circ} 13.467$, si tous les membres d'une catégorie bénéficient des acquis de la négociation collective, le syndicat ne peut exiger aucune forme de cotisation de la part des travailleurs qui ne sont pas adhérents d'un syndicat. Dans ce cas, et toujours selon la loi $n^{\circ} 13.467$, le syndicat aurait besoin du consentement de chaque salarié appartenant à la catégorie pour percevoir une cotisation. Cette situation peut encourager les «resquilleurs", c'est-à-dire ceux qui bénéficient des effets de la négociation collective, mais ne paient pas les coûts engagés par le syndicat lors de la négociation.

\section{I - DES COTISATIONS SYNDICALES OBLIGATOIRES DEVENUES FACULTATIVES: LES MODIFICATIONS DES RÈGLES}

L'un des principaux éléments du système corporatiste brésilien était l'imposition par la loi de cotisations syndicales universelles, collectées auprès des employeurs et des travailleurs représentés par des syndicats. Dans ce système, il existait essentiellement quatre types de cotisations syndicales: les cotisations versées individuellement par les

1 Action d'inconstitutionnalité directe \#5794:

http://portal.stf.jus.br/processos/detalhe.asp?incidente $=5288954$

2 Dans le système corporatiste brésilien, les employeurs s'organisent également en syndicats regroupés par catégories économiques. Ici, nous ne nous intéresserons qu'aux syndicats de travailleurs. 
travailleurs qui choisissent d'adhérer à un syndicat (CLT, article 548, b), les cotisations négociées (basées sur une disposition générale de l'article 513 de la CLT), les cotisations confédérales (article 8, IV, Constitution fédérale), et les cotisations syndicales prévues par la loi et à la charge de tous les membres dıune catégorie (articles 578 à 610 de la CLT). Seul le dernier type de cotisations imposées par la CLT était obligatoire pour tous les travailleurs ou employeurs d'une catégorie.

Les cotisations syndicales universelles prévues par la CLT constituaient une part importante des ressources des syndicats, notammentceux de petite taille quien dépendaient presque entièrement. Perçues une fois par an, ces cotisations étaient obligatoires pour les travailleurs salariés, syndiqués ou non, les travailleurs indépendants et les employeurs dans le cas des syndicats d'employeurs. La valeur de cette cotisation avoisinait une journée de salaire dans le cas des syndicats de travailleurs ${ }^{3}$.

Or les nouvelles règles rendent ces cotisations syndicales facultatives, c'est-à-dire que leur paiement est soumis à l'acceptation formelle et individuelle des travailleurs.

Ce changement a provoqué une baisse importante des cotisations qui assuraient la survie économique des syndicats. Les données de l'ancien ministère du Travail ${ }^{4}$ indiquent que le montant lié aux cotisations syndicales prévues par la CLT et versées aux syndicats a diminué de $90 \%$ en $2018^{5}$.

La fin des cotisations syndicales obligatoires a été considérée comme un moyen de rendre les syndicats brésiliens plus représentatifs, en les obligeant à avoir un contact direct avec leurs membres. La réaction des syndicats aux changements de 2017 a pris principalement la forme d'une relance de la cotisation négociée, sur la base de l'article 513 de la CLT. La cotisation est fixée par une assemblée de travailleurs, qui définit alors le pourcentage à percevoir, le prélèvement de la cotisation étant prévu dans une convention collective et effectué par l'employeur sur les salaires de tous les travailleurs de la même catégorie. La question est maintenant de savoir si la cotisation négociée est constitutionnelle, compte tenu des positions jurisprudentielles avant et après la réforme, et notamment de la décision de la Cour suprême sur la constitutionnalité de la loi $n^{\circ} 13.467$ de 2017 rendant facultatives les cotisations syndicales CLT.

\section{II - LA COTISATION NÉGOCIÉE ET LA LIBERTÉ D'ASSOCIATION NÉGATIVE}

Les syndicats intègrent déjà la cotisation négociée dans les conventions collectives ${ }^{6}$. Cette solution se heurte à la jurisprudence précédant la loi nº 13.467 de 2017 . Avant cette loi, dans le cas des cotisations confédérales et négociées, les tribunaux considéraient le

3 Le montant total en 2015 s'élevait à environ 1 milliard de dollars US pour les syndicats de travailleurs et d'employeurs. Ce n'est qu'en 2015 que le Ministère du Travail a été obligé de divulguer ces sommes: http://www.cgu.gov.br/noticias/2015/07/cidadaos-usam-lei-de-acesso-para-obter-receitade-sindicatos-com-contrib uicao-obrigatoria

4 Le $1^{\text {er }}$ janvier 2019, un décret présidentiel intitulé «la mesure provisoire 870 » a supprimé le ministère du Travail (fédéral), en répartissant ses fonctions entre trois ministères.

5 Journal O Estado de San Paulo: https://economia.estadao.com.br/noticias/geral,sindicatosperdem-90-da-contribuicao-sindical-no-1-ano-da-reforma-trabalhista,70002743950

6 Voir Acordo Coletivo de Trabalho 2018/2018, CE000016/2018, 18/01/2018: http://www3.mte. gov.br/sistemas/mediador/ 
versement obligatoire des cotisations comme étant contraire au principe de la liberté syndicale, même si les cotisations étaient approuvées par l'assemblée des travailleurs et négociées avec l'employeur.

Après la promulgation de la loi n 13.467 de 2017, des actions en justice présentées devant la STF font valoir que la Constitution fédérale autoriserait les cotisations syndicales obligatoires. Le 29 juin 2018, dans I'une de ces affaires (action d'inconstitutionnalité directe $n^{\circ} 5794$ ), la STF a jugé que les articles 545, 578, 579, 582, 582, 583, 587 et 602 de la CLT, qui supprimaient le caractère obligatoire des cotisations syndicales, étaient bien constitutionnels. On peut citer le Ministre Alexandre de Moraes: «II n’y a pas d'autonomie tant qu'un système syndical dépend de l'argent de l'Etat pour survivre ». L'élément central de cette discussion est de savoir si la Cour suprême continue à adopter le même raisonnement qu'avant la loi n 13.467 de 2017 en ce qui concerne l'incompatibilité de la collecte de cotisations syndicales auprès de tous les membres d'une catégorie - adhérents ou non - et le point V de l'article 8 de la Constitution : «V. : Nul ne sera obligé de s'affilier ou de se maintenir affilié à un syndicat ».

\section{III - UNE NOUVELLE COTISATION BASÉE SUR LE PRINCIPE DE LA LIBERTÉ D'ASSOCIATION}

Le débat autour de cette question implique l'interprétation de deux règles de la CLT: l'article 513, alinéa e, et l'article 611-B, point XXVI, inclus dans la loi n 13.467 de 2017. L'article 513, alinéa e, autorise un syndicat à imposer une cotisation à tous les membres d'une catégorie, ce qui constitue, indiqué précédemment, la base juridique de la cotisation négociée. Conformément au principe de la liberté d'association, cette cotisation serait idéalement établie par le biais d'une assemblée de travailleurs, convoquée à cet effet, qui voterait pour fixer la valeur de la cotisation et formaliser le consentement de la catégorie en question.

Ce modèle serait conforme au principe de la liberté syndicale exprimé par la Convention $n^{\circ} 87$ de l'Organisation Internationale du Travail (OIT), qui n'interdit pas les cotisations imposées aux travailleurs non membres d'un syndicat, à condition que ces cotisations soient décidées par le biais d'une délibération démocratique au sein du syndicat. Dans les systèmes où les syndicats représentent les travailleurs adhérents ou non au syndicat, comme c'est le cas au Brésil, il est logique d'imposer des cotisations aux travailleurs non affiliés. La cotisation doit alors être décidée par les travailleurs eux-mêmes, au sein de l'assemblée, et sa collecte doit être prévue par une négociation collective. Les anciennes cotisations syndicales enfreignaient le principe de la liberté syndicale puisqu'elles étaient imposées par la loi à l'ensemble d'une catégorie et que leur montant était déterminé par la loi sans la participation appropriée des membres de cette catégorie.

S'agissant du point XXVI de l'article 611-B de la CLT, inclus dans la loi $n^{\circ} 13.467$ de 2017, il interdit la négociation collective sur l'établissement de cotisations obligatoires pour l'ensemble d'une catégorie.

Force est de constater qu'une telle limitation enfreint le principe de la liberté syndicale plutôt que de le protéger, même si les tribunaux du travail semblent avoir tendance à conclure que la perception de cotisations auprès des travailleurs non-syndiqués porte 
atteinte à la liberté d'association négative7. A cet égard, le Procureur fédéral du travail a exprimé sa position dans une note technique de 2018, faisant valoir que «la Constitution n'interdit pas la clause de sécurité syndicale», c'est-à-dire des clauses "permettant de percevoir les cotisations de non-adhérents, à condition qu'ils soient couverts par la convention collective », car ce type de cotisation ne viole pas le point $\mathrm{V}$ de l'article 8 de la Constitution ».

Lorsqu'on évoque la violation éventuelle de l'article $8(V)$ de la Constitution par une clause de sécurité syndicale, le premier point à examiner est de savoir si une telle clause était prévue par la loi ou par la négociation collective. Des cotisations syndicales prévues par la loi constitueraient une restriction au principe de la liberté syndicale, à l'instar de l'ancien article 578 de la CLT sur les cotisations syndicales. Les cotisations syndicales établies en vertu d'une clause de sécurité syndicale dans les négociations collectives ont pour effet de renforcer le pouvoir des syndicats en étendant aux travailleurs non adhérents (et qui peuvent le rester) les avantages découlant des négociations collectives.

Renforçant le statut différencié de la négociation collective, l'article 8 de la Convention $n^{\circ} 95$ de l'OIT sur la protection du salaire, ratifiée par le Brésil en 1957, stipule que « des retenues sur les salaires ne seront autorisées que dans des conditions et limites prescrites par la législation nationale ou fixées par une convention collective ou une sentence arbitrale ».

La réforme apportée par la loi n 13.467 de 2017 se justifie dans la mesure où elle vise à garantir une plus grande latitude à la négociation collective. Ses règles imposent toutefois des limitations à la négociation collective qui ne reposent pas sur des valeurs de l'ordre du travail. Le point XXVI de l'article 611 b en est un exemple. La liberté d'association, garantie dans le libellé de l'article 8 de la Constitution et dans son point V, n'interdit pas la négociation collective de cotisations obligatoires pour une catégorie donnée, si ces cotisations sont justifiées par un système de représentation par catégorie et l'exclusivité de la représentation, en vertu du point II de l'article 8 . La loi ne peut donc pas restreindre la liberté d'association et le droit de négociation collective, en violation de la Constitution fédérale et, selon l'interprétation de l'OlT elle-même, en violation des Conventions $n^{\circ} 87$ et 98 de l'OIT ratifiées par le Brésil.

7 Voir http://www.trt7.jus.br/files/publicacoes/Enunciados aprovados 2018.pdf 